\title{
Precision Electron Beam Polarimetry
}

W. Lorenzon

Citation: AIP Conference Proceedings 1149, 709 (2009); doi: 10.1063/1.3215743

View online: https://doi.org/10.1063/1.3215743

View Table of Contents: http://aip.scitation.org/toc/apc/1149/1

Published by the American Institute of Physics

\section{Conference Proceedings}

\section{Get $30 \%$ off all print proceedings!}

Enter Promotion Code PDF-30 at check 


\title{
Precision Electron Beam Polarimetry
}

\author{
W. Lorenzon \\ Randall Laboratory of Physics, University of Michigan, Ann Arbor, Michigan 48109-1040, USA
}

\begin{abstract}
Measurements at future electron accelerators will require unprecedented precision in the determination of electron beam polarization. Existing and novel electron beam polarization measurement schemes are examined, and ideas are being presented of how to to achieve sub- $1 \%$ precision in polarization determination.
\end{abstract}

\section{INTRODUCTION}

In order to reach the physics goals at future electron/positron accelerators such as the Electron Ion Collider (EIC) or the International Linear Collider (ILC), strong requirements are placed on precision polarimetry for electrons and positrons. The current design of the EIC project foresees collisions of 3-20 GeV longitudinally polarized electrons on $30-250 \mathrm{GeV}$ protons or 50-100 GeV/u heavy ions (such as gold) with bunch separations of 3-35 ns. It is anticipated that the electron beam polarization is $70 \%$ or better and that it needs to be measured with high precision ( $\lesssim 1 \%$ systematic uncertainty). The current design of the ILC project foresees collisions of $45.6-500 \mathrm{GeV}$ longitudinally polarized electrons and positrons. It is anticipated that the electron (positron) beam polarization is $80 \%(50 \%)$ or better and that it needs to be measured with unprecedented precision ( $\lesssim 0.25 \%$ systematic uncertainty). This is a factor of two better than ever achieved before, and thus raises the question whether this is a realistic goal. Before trying to address this question, it is advantageous to take a step back and consider what has previously been achieved in precision beam polarimetry.

There are many polarimeters that have been in use, are in use, or are planned at various laboratories. Table 1 shows an overview of polarimeters that are or have been in use, and their precision [1]. The systematic uncertainties in beam polarization measurements for Compton polarimeters are reported to be in the $0.5-2 \%$ range, but they can get larger as measurements get pushed to lower beam energies $\left(E_{b} \lesssim 1.0 \mathrm{GeV}\right)$. For Møller scattering the systematic uncertainties are typically $2-3 \%$, and may approach $1 \%$ or below at high magnetic fields.

Although the precision of the JLab polarimeters, listed in Table 1, do not reach the precision required by the EIC nor the ILC, they still serve a valuable lesson in understanding the ultimate precision reachable in polarization measurements. The systematic uncertainties of the various polarimeters in the three experimental halls at JLab were each evaluated individually. Since it was possible to compare the polarization of the five polarimters with a special arrangement of the CEBAF accelerator, the "Spin Dance" Experiment was performed in July 2000 [2]. In this experiment, a multi-hall cross-normalization of the relative analyzing power of the five JLab electron polarime-

CP1149, Spin Physics, $18^{\text {th }}$ International Spin Physics Symposium

edited by D. G. Crabb, D. B. Day, S. Liuti, X. Zheng, M. Poelker, and Y. Prok

(C) 2009 American Institute of Physics 978-0-7354-0686-5/09/\$25.00 
TABLE 1. Overview of polarimeters that are or have been in use, and their precision

\begin{tabular}{|c|c|c|c|}
\hline Laboratory & Polarimeter & Relative precision & Dominant systematic uncertainty \\
\hline JLab & $\begin{array}{l}5 \mathrm{MeV} \text { Mott } \\
\text { Hall A Møller } \\
\text { Hall B Møller } \\
\text { Hall C Møller } \\
\text { Hall A Compton }\end{array}$ & $\begin{array}{l}\sim 1 \% \\
\sim 2-3 \% \\
1.6 \%(\rightarrow 2-3 \%)^{*} \\
0.5 \%(\rightarrow 1.3 \%)^{\dagger} \\
1 \%(@>3 \mathrm{GeV})\end{array}$ & $\begin{array}{l}\text { Sherman function } \\
\text { target polarization } \\
\text { target polarization, Levchuk effect } \\
\text { target polarization, Levchuk effect, } \\
\text { high current extrapolation } \\
\text { detector acceptance + response }\end{array}$ \\
\hline HERA & $\begin{array}{l}\text { LPol Compton } \\
\text { TPol Compton } \\
\text { Cavity LPol Compton } \\
\end{array}$ & $\begin{array}{l}1.6 \% \\
3.1 \% \\
? \\
\end{array}$ & $\begin{array}{l}\text { analyzing power } \\
\text { focus correction }+ \text { analyzing power } \\
\text { still unknown }\end{array}$ \\
\hline MIT-Bates & $\begin{array}{l}\text { Mott } \\
\text { Transmission } \\
\text { Compton }\end{array}$ & $\begin{array}{l}\sim 3 \% \\
>4 \% \\
4 \%\end{array}$ & $\begin{array}{l}\text { Sherman function }+ \text { detector response } \\
\text { analyzing power } \\
\text { analyzing power }\end{array}$ \\
\hline SLAC & Compton & $0.5 \%$ & analyzing power \\
\hline
\end{tabular}

ters was performed. The purpose of this comparison between the Mott, Compton, and Møller polarimeters was to reveal possible differences between the polarimeters that are systematic in nature and have not previously been accounted for. The results are displayed in Fig. 1 with the solid symbols. There is significant discrepancy between the polarimeters, even if the systematic uncertainties are included.

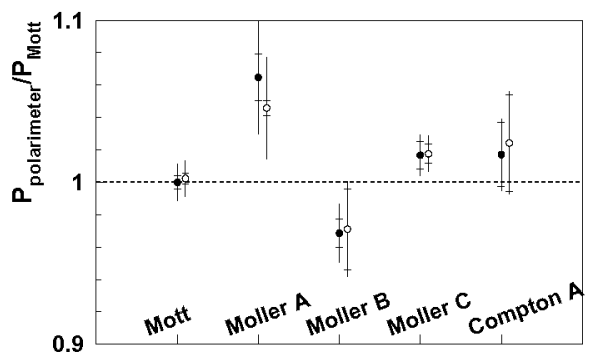

FIGURE 1. Relative analyzing power for the five JLab electron beam polarimeters, normalized to the Mott polarimeter for comparison. The solid symbols are the results for the entire data set. The open symbols represent the results for the data set limited to be within $25 \%$ of the maximum measured polarization.

Since the Hall A and B Møller polarimeters may have systematic effects that depend on the transverse components of the electron beam polarization, which are large when the longitudinal components are small, the data shown in open symbols have been restricted to be within $25 \%$ of the maximum polarization value. These results indicate that the horizontal component of polarization may be an important source of systematic effects for the Hall A Møller polarimeter. For the reduced data set, the discrepancy among the five polarimeters becomes less significant. As a result of the Spin Dance Experiment, the Hall A Møller polarimeter is implementing a Hall C style target to be able to isolate instrumental from target polarization effects. 


\section{LESSONS LEARNED}

Many lessons have been learned from these earlier polarization measurements. Experience at many laboratories has taught us that it is imperative to include polarization diagnostics and monitoring capabilities in the design of the beam lattice. It is important to ensure that the beam polarization can be measured continuously during data taking to minimize systematic uncertainties associated with the beam polarization, such as drifts or luminosity related variations in polarization. If at all possible, the beam polarization should be measured at the IP, or as close to the IP as possible. The laser and beam polarizations have to be flipped at intervals that are short compared to any drifts in polarization. The cross-comparison of the analyzing power of various polarimeters at JLab has shown that providing or even proving precision at the $1 \%$ level is challenging. Since the requirements at future electron/positron accelerators are even stricter, it is absolutely crucial that multiple devices are employed for testing the systematic uncertainties of each polarimeter. There has to be at least one technique that can measure the absolute polarization of the beam, while others can do relative measurements.

Further, Compton scattering appears to be the ideal process for measuring the polarization of high energy, high beam current electron (positron) beams. Compton scattering is a pure QED process where no atomic or nuclear corrections have to be applied, and where radiative correction uncertainties are at the $0.1 \%$ level [3]. In Compton scattering, the target (i.e. laser beam) is effectively 100\% polarized while in Møller scattering the target (i.e. polarized electrons in iron foils) is only about $8 \%$ polarized. Furthermore, Compton scattering measurements are non-invasive, while Møller scattering measurements are destructive due the need of using iron or iron-alloy foils. Compton scattering is ideal for high beam currents, while Møller scattering measurements suffer from beam induced foil heating effects at beam currents above a few $\mu \mathrm{A}$.

To achieve sub-1\% precision in the electron (positron) beam polarization determination, all these consideration have to be taken into account, and if possible, new and innovative ideas have to be employed.

\section{NEW IDEAS FOR FUTURE ELECTRON ACCELERATORS}

Most of the major disadvantages of Møller scattering might be overcome with a new idea that employs polarized atomic hydrogen in an ultra-cold magnetic trap [4]. It is argued that at $300 \mathrm{mK}$, the electrons of the hydrogen atoms are brute-force polarized to $100 \%$ within a factor of $10^{-3}$, and a polarization measurement with a statistical uncertainty of $1 \%$ can be achieved in 10 min with a beam current of $100 \mu \mathrm{A}$ and a target density of $3 \times 10^{15} \mathrm{~cm}^{-3}$. Employing an atomic hydrogen target has the advantage that it is noninvasive and can be used to continuously measure the beam polarization, and that it may provide a systematic uncertainty below $0.5 \%$. It has the disadvantage, though, that the target is very complex, and that gas heating effects by radiation grow with the beam intensity squared. This might be a serious limitation for the high currents envisioned for the EIC or ILC. One solution around the gas heating effect might be to consider a hydrogen jet target instead. Further studies are underway to explore this interesting idea. 
New developments in laser technology might give a big boost to Compton scattering based polarization measurements, where it has been necessary to build either delicate laser cavity lasers or use high power pulsed lasers to get Compton rates that allow polarization measurements within reasonable time scales. This technology is being borrowed from fiber based drive lasers at electron sources that provide very high power, and use gain switching, as compared to mode locking which is sensitive to mode lock problems. The advantages are that they can be phase locked to the actual beam of the accelerator, therefore providing a nearly $100 \%$ duty cycle. In addition, fiber lasers can be easily accessed since they are external to the beam line vacuum system (unlike cavity lasers). They further provide excellent stability, low maintenance, and straightforward implementation. Efforts are underway to build a Compton polarimeter using the fiber lasers for a new Hall C Compton polarimeter.

There is general agreement that detection of Compton electrons is the best tool for high precision polarimetry. Since the analyzing power depends strongly on the momentum of the Compton electrons, Compton electrons are typically analyzed by fitting the asymmetry shape over parts or the entire available momentum range. Alternatively, the Compton edge (which corresponds to the minimum energy of the back-scattered Compton electrons), can be used to determine the electron beam polarization. These methods however depend strongly on the response function of the detector, which must be calibrated and monitored carefully. A new idea to do a zero-crossing Compton electron analysis is suggested. It relies on the well-defined energies of the zero crossing of the asymmetry (corresponding to $90^{\circ}$ scattering in the electron rest frame) and of the Compton edge. This analysis is based on a linear fit of the zero crossing of the Compton asymmetry, and an integration of the asymmetry spectrum from that point to the Compton edge, instead of a fit to the spectrum shape between those points. It has the advantage that no absolute energy response calibration of the detector is necessary, and that the corrections due to finite detector position and energy resolutions are small $(\ll 1 \%)$.

\section{Polarimetry at the EIC}

A rough idea for a polarimeter suitable to withstand the high luminosity at EIC is shown in Fig. 2. A chicane is introduced to separate Compton polarimetry from the electron beam. This introduces ample space for Compton photon detection, and a convenient way to separate the Compton electrons from the beam electrons in one of the soft bending magnets of the chicane. The chicane helps to minimize background rates: minimizing bremsstrahlung background requires to have a short section of beam line, while minimizing synchrotron background requires a chicane with soft bends. The Compton photon detector can be operated in counting and integrating mode. A pair spectrometer consisting of a variable converter (to select the appropriate rate of $e^{+} e^{-}$pairs in counting mode), a dipole magnet (to separate the pair-produced electrons and positrons), and position sensitive detectors could be configured for photon counting. Downstream of the pair spectrometer a radiation hard and fast $(<35 \mathrm{~ns})$ position sensitive sampling calorimeter could be used to operate in integrating mode. If appropriate photomultiplier tubes are used, the sampling calorimeter can be operated in counting and integrating mode. 


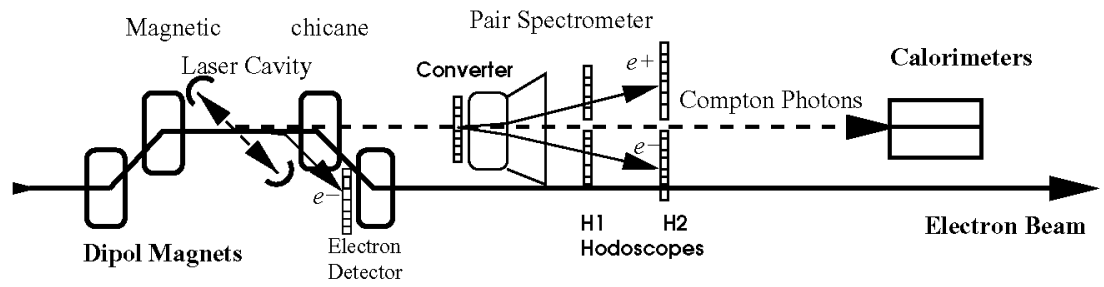

FIGURE 2. Schematic view of a possible Compton polarimeter for the EIC.

\section{Polarimetry at the ILC}

The current scheme for polarimetry at the ILC proposes three ways to measure polarization: an upstream Compton polarimeter (1.8 km from IP) shown in Fig. 3, a downstream Compton polarimeter (150 m from IP) and the $e^{+} e^{-} \rightarrow W^{+} W^{-}$process which is measured at the IP and which is very sensitive to electron and positron polarization (at the $0.1 \%$ level). There is however some significant complication in this scheme. The polarization at the IP is the luminosity-weighted polarization which is not identical to the beam polarization at the upstream polarimeter. Furthermore, although the downstream polarimeter measures the luminosity-weighted polarization, there are depolarization and spin transport effects that are estimated to be at the 0.1-0.4\% levels. Those effects are relatively large and of the same magnitude as the required accuracy, which makes it imperative that the uncertainties in these effects are well understood and kept small.

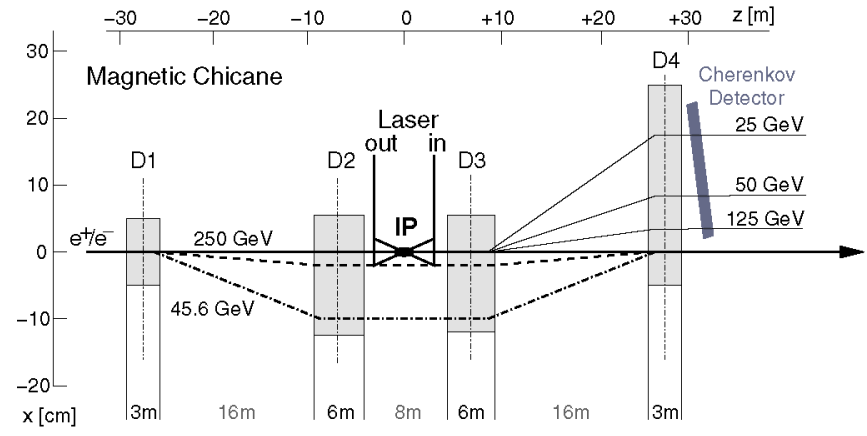

FIGURE 3. Schematic view of a possible upstream Compton polarimeter for the ILC.

In order to make a convincing case for a $0.25 \%$ precision in electron and positron polarimetry, all three techniques are needed. It is necessary to determine the most accurate polarization value for each polarimeter separately. It is then important to make sure that all depolarization and spin transport effects are understood. Only at this point the values for the up- and downstream polarimeters should be compared. If everything is understood properly, they should agree. The final calibration of the absolute polarization scale should be performed with the with $e^{+} e^{-} \rightarrow W^{+} W^{-}$process. Only if all three measurements are in agreement, good confidence can be gained that the required precision has been achieved. 


\section{Summary}

In summary, it appears that electron beam polarimetry can be measured with high precision. There are no serious obstacles to achieve a precision of $1 \%$. It is imperative though to include polarimetry in the beam lattice and the interaction region design. And it is crucial to use multiple devices and even techniques to control the systematic uncertainties at the sub-1\% level.

Reaching a precision of $0.25 \%$ presents a big challenge, but there are no apparent show stoppers. The large beam energy at the ILC is crucial, since it provides analyzing powers in Compton scattering that approach unity. Reaching a precision of $0.1 \%$ in contrast enters entirely new territory. This is well beyond traditional polarimetry and requires to utilize specific physics processes that are very sensitive to polarization.

There are many issues that require careful scrutiny. The beam crossing frequency is proposed to be somewhere between 3-35 ns at the EIC. This is very different from the crossing frequencies at RHIC (106 ns) and HERA (96 ns). HERA has demonstrated that beam-beam induced depolarization becomes important at high luminosities. An entirely new concept for the EIC is crab-crossing of bunches. What effect will it have on the beam polarization, and how can these effects be measured? Finally, depolarization and spin transport effects need to be understood to high accuracy at the ILC.

To tackle these issues, it will require a group of experienced physicists who can build on their experience, and at the same time are open to new developments in the field. With much work already done, extensive modeling will be necessary to optimize the design (i.e. analyzing power calibration, modeling of beam depolarization and spin transport, etc.) for polarimetry at a future electron/positron accelerator.

\section{ACKNOWLEDGMENTS}

The author's research is supported in part by the U.S. National Science Foundation, Intermediate Energy Nuclear Science Division under grant No. PHY-0555423. The author thanks Eugene Chudakov, Wouter Deconinck, Dave Gaskell, Avetik Hayrapetyan, Jenny List, Jeff Martin, and Kent Paschke for many fruitful discussions and for crucial contributions to this paper.

\section{REFERENCES}

1. Lorenzon W., "Precision Electron Beam Polarimetry", AIP Conference Proceedings No. 980, ed. A. Kponou, Y. Makdisi, A. Zelenski p. 407 (2008).

2. Grames, J. M. et al., Phys. Rev. ST Accel. Beams 4, 042802 (2004).

3. Swartz M.L., et al., Phys. Rev. D58, 014010 (1998).

4. Chudakov, E. A., and Luppov, V. G., IEEE Trans. Nucl. Sci. 51, 1533 (2004). 